\title{
Structural Brain Imaging in Children and Adolescents following Prenatal Cocaine Exposure: Preliminary Longitudinal Findings
}

\author{
Nurunisa Akyuz ${ }^{a-c}$ Minal V. Kekatpure ${ }^{a, b}$ Jie Liu ${ }^{d}$ Stephen J. Sheinkopf ${ }^{d}$ \\ Brian T. Quinn ${ }^{a, b}$ Meenakshi D. Lala ${ }^{a, b}$ David Kennedy ${ }^{e}$ Nikos Makris ${ }^{e}$ \\ Barry M. Lesterd Barry E. Kosofsky ${ }^{a, b}$ \\ Departments of a Neurology and Neuroscience, ${ }^{b}$ Pediatrics and ${ }^{\mathrm{c} P h y}$ siology and Biophysics, Weill Cornell Medical \\ College, New York, N.Y., ${ }^{d}$ Center for the Study of Children at Risk, Warren Alpert Medical School of Brown University \\ Women and Infants' Hospital, Providence, R.I., and e'Center for Morphometric Analysis, Massachusetts General \\ Hospital, Charlestown, Mass., USA
}

\section{Key Words}

Structural brain imaging - Prenatal cocaine exposure . Developing brain - Magnetic resonance imaging - Prenatal cocaine

\begin{abstract}
The brain morphometry of 21 children, who were followed from birth and underwent structural brain magnetic resonance imaging at 8-10 years, was studied. This cohort included 11 children with prenatal cocaine exposure (CE) and 10 noncocaine-exposed children (NCE). We compared the CE versus NCE groups using FreeSurfer to automatically segment and quantify the volume of individual brain structures. In addition, we created a pediatric atlas specifically for this population and demonstrate the enhanced accuracy of this approach. We found an overall trend towards smaller brain volumes among CE children. The volume differences were significant for cortical gray matter, the thalamus and the putamen. Here, reductions in thalamic and putaminal volumes showed a robust inverse correlation with exposure levels,
\end{abstract}

thus highlighting effects on dopamine-rich brain regions that form key components of brain circuitry known to play important roles in behavior and attention. Interestingly, head circumferences (HCs) at birth as well as at the time of imaging showed a tendency for smaller size among CE children. HCs at the time of imaging correlated well with the cortical volumes for all subjects. In contrast, $\mathrm{HCs}$ at birth were predictive of the cortical volume only for the CE group. A subgroup of these subjects ( $6 \mathrm{CE}, 4 \mathrm{NCE})$ was also scanned at 13-15 years of age. In subjects who were scanned twice, we found that the trend for smaller structures continued into teenage years. We found that the differences in structural volumes between the CE and NCE groups are largely diminished when the HCs are controlled for or matched by study design. Participants in this study were drawn from a unique longitudinal cohort and, while the small sample size precludes strong conclusions regarding the longitudinal findings reported, the results point to reductions in $\mathrm{HCs}$ and in specific brain structures that persist through teenage years in children who were exposed to cocaine in utero.

(c) 2014 S. Karger AG, Basel

\section{KARGER}

E-Mail karger@karger.com

www.karger.com/dne
C 2014 S. Karger AG, Basel

0378-5866/14/0364-0316\$39.50/0
Barry E. Kosofsky, MD, PhD

Division of Child Neurology, Weill Cornell Medical College

New York Presbyterian Hospital, 525 East 68th Street, Box 91

New York, NY 10021 (USA)

E-Mail bar2009@med.cornell.edu 


\section{Introduction}

A large number of studies have provided accumulating evidence that prenatal exposure to cocaine may have long-term detrimental effects on the developing brain [13]. Although cocaine has many global effects on brain physiology, including constriction of blood vessels [4], its long-term effects on brain development have been largely linked to its action on monoamine neurotransmitters. Importantly, cocaine can readily cross the placenta and the blood-brain barrier to reach binding sites on monoamine transporters on neurons in the fetal brain [5-7]. By binding to and blocking the activity of these membrane transporters, including dopamine, serotonin (5-HT), and to a lesser extent norepinephrine transporters [8-10], cocaine can directly interfere with the development of these neurotransmitter systems, with long-term effects on their development and subsequent function [11-13]. Correspondingly, animal studies have associated prenatal cocaine exposure (CE) with alterations in brain development, particularly in dopamine-rich brain regions [1416]. Clinical studies have reported prenatal cocaine-induced reductions in weight and head circumferences (HCs) at birth [17-19], as well as subsequent effects on behavior and cognitive functioning that are subtle but significant [20-22]. Specifically, prenatal CE has been associated with low birth weight (small for gestational age) and smaller HCs at birth [23-25], as well as at age 10-14 years [26] - consistent with a direct effect in altering fetal and postnatal brain growth.

In order to explore whether CE in utero leads to longterm alterations in volumetric brain growth, that may be evident globally or restricted to particularly vulnerable structures, we performed structural magnetic resonance imaging (MRI) on CE and noncocaine-exposed (NCE) children. This project was part of the multisite Maternal Lifestyle Study (MLS) with study centers in Providence, R.I., Miami, Fla., Memphis, Tenn. and Detroit, Mich., USA [27], tracking cohorts of prenatally drug-exposed children and matched controls from birth to adolescence. Here, we report regional patterns of brain morphometry in a group of CE versus NCE children (age range 8-10 years) and compare these results with those we have previously published during adolescence (age range 13-15 years) $[28,29]$. We hypothesize that structural markers of altered brain development may yield valuable clinical markers for assessment, monitoring and treatment of prenatally drug-exposed offspring.

To date, neuroimaging studies from our and other groups have collectively reported a tendency for smaller cortical and subcortical structures, including a significantly smaller caudate $[26,30]$, corpus callosum [31], pallidum [28] and caudate nucleus [32], with the exception of a larger amygdala [30] in CE children - all in agreement with the notion that prenatal CE may have longterm detrimental effects on brain growth. Here, we report the use of a pediatric brain atlas customized for use with the FreeSurfer software package that we developed based on a training data set from 14 children ( 8 with prenatal CE, 2 with polysubstance exposure and 4 controls) and show the improved effectiveness of using this case- and age-specific atlas in segmenting these pediatric brains to that of using the standard adult atlas. Second, we used our atlas to quantify pediatric brain morphometry in order to determine which brain structures were specifically affected as a consequence of prenatal CE. We relate the structural findings to clinical variables and, finally, we interpret our results in the context of our previously published study performed on an overlapping set of MLS subjects studied at $13-15$ years of age [28].

\section{Materials and Methods}

\section{Subjects}

The study sample is drawn from the MLS, a longitudinal project characterizing the development of children who were prenatally exposed to cocaine and other drugs of abuse [27]. Prenatal exposure to cocaine and opiate was identified by meconium toxicology (positive enzyme multiple immunoassay test, EMIT) followed by gas chromatography/mass spectroscopy confirmation. Infants were entered into the comparison group if there was a negative EMIT screen for cocaine and opiate metabolites. Exclusion criteria included chromosomal abnormalities, TORCH (toxoplasmosis, rubella, cytomegalovirus, herpes, syphilis) group of infections confirmed prior to 1 month of the infant's corrected postnatal age, or relocation outside the catchment area. Additional information on the frequency of use of prenatal cocaine and other substances during pregnancy was determined by interview with mothers at 1 month of the infant's corrected postnatal age using the Maternal Inventory of Substance Use [33]. Prenatal maternal use of cigarettes, alcohol and marijuana was present in both groups. Dyads were classified into 3 groups: no prenatal exposure, some exposure ( $<3$ days per week during the first trimester) and heavy exposure (defined as reported cocaine use of $\geq 3$ days per week during the first trimester) [27].

The MLS was conducted at four different study sites, including Brown University/Women and Infants' Hospital, the University of Miami, the University of Tennessee, Memphis and Wayne State University. A 'certificate of confidentiality' from the US Department of Health and Human Services allowed the study to maintain participant confidentiality with regard to drug use information. The certificate of confidentiality applied specifically to information regarding maternal use of illegal drugs and left in force all reporting requirements with regard to child abuse or child neglect. 
Participants were fully informed of their rights and limits as study participants. Limits to confidentiality and informed consent were obtained from all caregivers, as approved by the review boards at each study site.

A total of 1,388 children were included in the MLS (658 CE, 730 NCE), with 211 enrolled at the Brown/Women and Infants' Hospital site (107 CE, $104 \mathrm{NCE}$ ). The original clinical cohort for this imaging study included 88 subjects recruited from the Brown/ Women and Infants' Hospital site and followed from birth to age 8-10 years, wherein the imaged subgroup was comprised of $13 \mathrm{CE}$ and 15 NCE subjects. The NCE group included 10 subjects with polysubstance exposure (without cocaine but including exposure to alcohol, tobacco and marijuana) and 5 subjects with no substance exposure, whose data were combined. Gestational age was determined by the obstetrician's best estimate; 1 of the NCE children was born before 33 weeks' gestational age. The HCs were recorded at 1 and 4 months following the infant's corrected postnatal age and then annually. Socioeconomic status was assessed using the Hollingshead index and modified to allow for application of the measure to nonnuclear families, while retaining the traditionally weighted educational and occupational scores. The study was approved by the appropriate institutional review boards at the Women and Infants' Hospital of Rhode Island, the Memorial Hospital of Rhode Island and Rhode Island Hospital. Informed consent was obtained from all parents or guardians, and all children provided assent.

A second cohort of 40 children (20 CE, 20 NCE) from the MLS participated in an imaging study at 13-15 years of age [28]; 10 children from this cohort ( $6 \mathrm{CE}, 4 \mathrm{NCE}$ ) participated in the imaging studies at both 8-10 and 13-15 years, forming a 5-year follow-up study of children recruited from the Providence site [28]. In the later study, the groups were designed to match based on maternal alcohol use, gender, IQ and socioeconomic status, and unlike the original study for HCs at birth [28]. This study was also approved by the relevant institutional review boards.

\section{MRI Acquisition}

All 28 children underwent MRI when they were 8-10 years old. Imaging was performed at the Memorial Hospital, a teaching hospital of Brown Medical School, on a 1.5-tesla Siemens Symphony with quantum gradients using a standard birdcage head coil. No sedation was used during image acquisition. Overall, 2 T1-weighted magnetization-prepared rapid gradient echo scans (MP-RAGE) were obtained from each participant with the following protocol: $\mathrm{TE}=4.1 \mathrm{~ms}, \mathrm{TR}=1,900 \mathrm{~ms}$, field of view $=256 \mathrm{~mm}$, slice thickness $=1 \mathrm{~mm}$, resolution $=1 \times 1 \times 1 \mathrm{~mm}$, gap $=0$, data matrix size $=$ $256 \times 256$ and inversion time $=1,100 \mathrm{~ms}$. Some of the scans $(2 \mathrm{CE}$, $5 \mathrm{NCE}$ ) were discarded due to motion artifacts. The follow-up study employed the same MP-RAGE pulse sequence on a 3-tesla Siemens TIM Trio scanner [28]. The acquisitions were averaged to increase the signal-to-noise ratio in all subjects who had 2 highquality scans. The high-quality scans were corrected for motion, normalized for intensity and skull-stripped using the FreeSurfer software package [34].

\section{Manual Subcortical Segmentation}

MRIs from 14 subjects were manually segmented at the Center for Morphometric Analysis, Massachusetts General Hospital. The technique for manually labeling brain structures has been previously validated and is based on contour mapping for each coronal plane [35-37]. The borders are based on signal intensity transitions at brain-CSF or gray-white matter interfaces as well as prior knowledge about the global positions of individual structures within the brain, their positions with respect to neighboring structures and various anatomical landmarks. The entire brain is divided into the cerebrum, the brain stem and the cerebellum. The cerebrum is further divided into the cerebral cortex, cerebral white matter, the thalamus, the caudate, the putamen, the pallidum, the hippocampus and the amygdala.

\section{Automated Subcortical Segmentation}

FreeSurfer software package version 4.0.5 (http://surfer.nmr. mgh.harvard.edu) using an automated pipeline custom developed for an XNAT-based DICOM server hosted at Weill Cornell Medical College of Cornell University (https://ped-birn.med.cornell. edu/xnat/) was used for the subcortical segmentation of the $3 \mathrm{D}$ MRIs. Details of this procedure have been described elsewhere [38]. In brief, this automated segmentation technique assigns a neuroanatomical label to each voxel on the image. The probability of each voxel being assigned a certain label depends on both subject-specific measures of image intensity and spatial information and subject-independent measures derived from a standard probabilistic atlas. Structures are labeled using an algorithm combining probabilities based on these measures and further spatial constraints imposed by computing the probability of a label occurring at a specific location given the neighboring labels, resulting in a segmentation with the same types of neighborhood relations as those observed in the manually labeled training set $[39,40]$. FreeSurfer segmentation employs a probabilistic atlas [41] based on a manually labeled training set generated from a set of 40 brain scans (10 young, 10 middle-aged, 10 elderly and 10 patients with $\mathrm{Alz}$ heimer's disease). The manual labeling approach was validated and used previously $[36,37,42]$. We developed and used an alternative atlas based on a manually labeled pediatric training set, using the same procedures as used in the creation of the standard FreeSurfer atlas [39] and subsequently comparing its accuracy with the results obtained from using the standard FreeSurfer atlas. Furthermore, this pediatric atlas included pediatric data sets from the prenatally substance-exposed group as well as the control group. This approach ensured that the structural characteristics from both groups were encoded in the atlas.

\section{Assessment of Validity of Using a Pediatric Atlas}

In order to assess the validity of using a new pediatric atlas to automatically label the subcortical structures, we adopted the jackknife/leave-one-out technique - a statistical resampling procedure $[43,44]$. Accordingly, from our original collection of 14 manual segmentations ( $8 \mathrm{CE}, 6 \mathrm{NCE}), 13$ of them were used as the training set for constructing the 'jackknife' atlas and automatically segment the left-out data set. This procedure was repeated until all 14 data sets were automatically segmented (fig. 1).

After the jackknife procedure was completed, we compared the automated and manual segmentations. This involved the following: (1) comparing the mean volume, (2) estimating the mean percent volume difference and (3) calculating the mean Dice similarity coefficients (DSC) [45] for each structure. The DSC is a measure of how much the automatically and manually segmented volumes overlap. Since the Dice measure is biased towards larger structures and yields no information regarding the spatial similarity of 2 segmentations in regions in which they do not overlap, we 

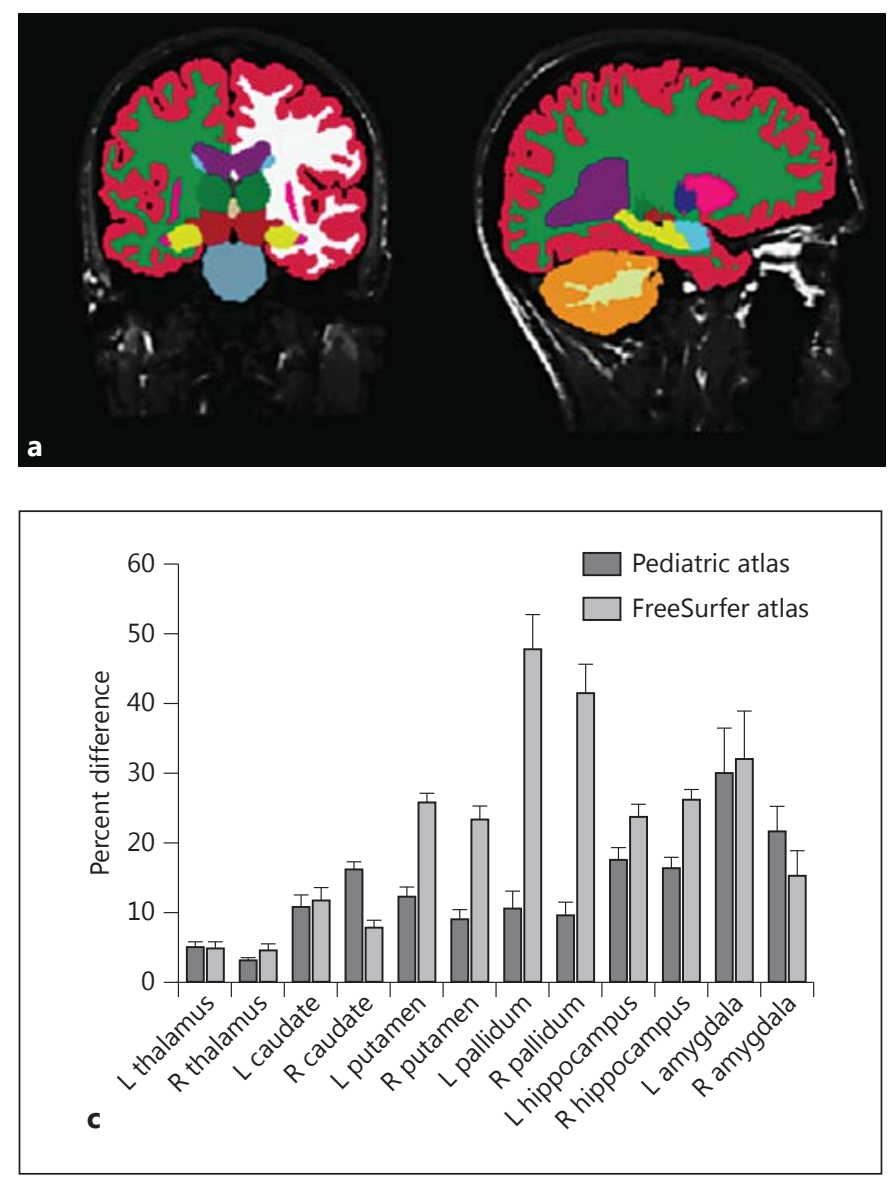

Fig. 1. Subcortical segmentation of the study data set using the pediatric atlas. $\mathrm{L}=$ left; $\mathrm{R}=$ right. a Coronal and sagittal views of segmented brain structures. Cortical gray matter, white matter and subcortical structures are represented in different colors. b The mean volumes and standard errors of subcortical structures as estimated using automated segmentation based on a pediatric atlas versus a standard FreeSurfer atlas, compared with those estimated

also computed a modified Hausdorff metric [46] that provides a measure of the spatial similarity of the 2 segmentations (e.g. manual and automated). Specifically, given a pair of segmentations, for each point on the boundary of each segmentation label, we computed the minimum distance to the boundary of the other segmentation and then averaged these distances.

\section{Statistical Analysis}

The brain MRIs of 21 subjects were automatically segmented using the pediatric atlas. Their HC measurements and demographic measures were also included in the data analysis. In order to determine the effects of prenatal CE, we divided the subjects into
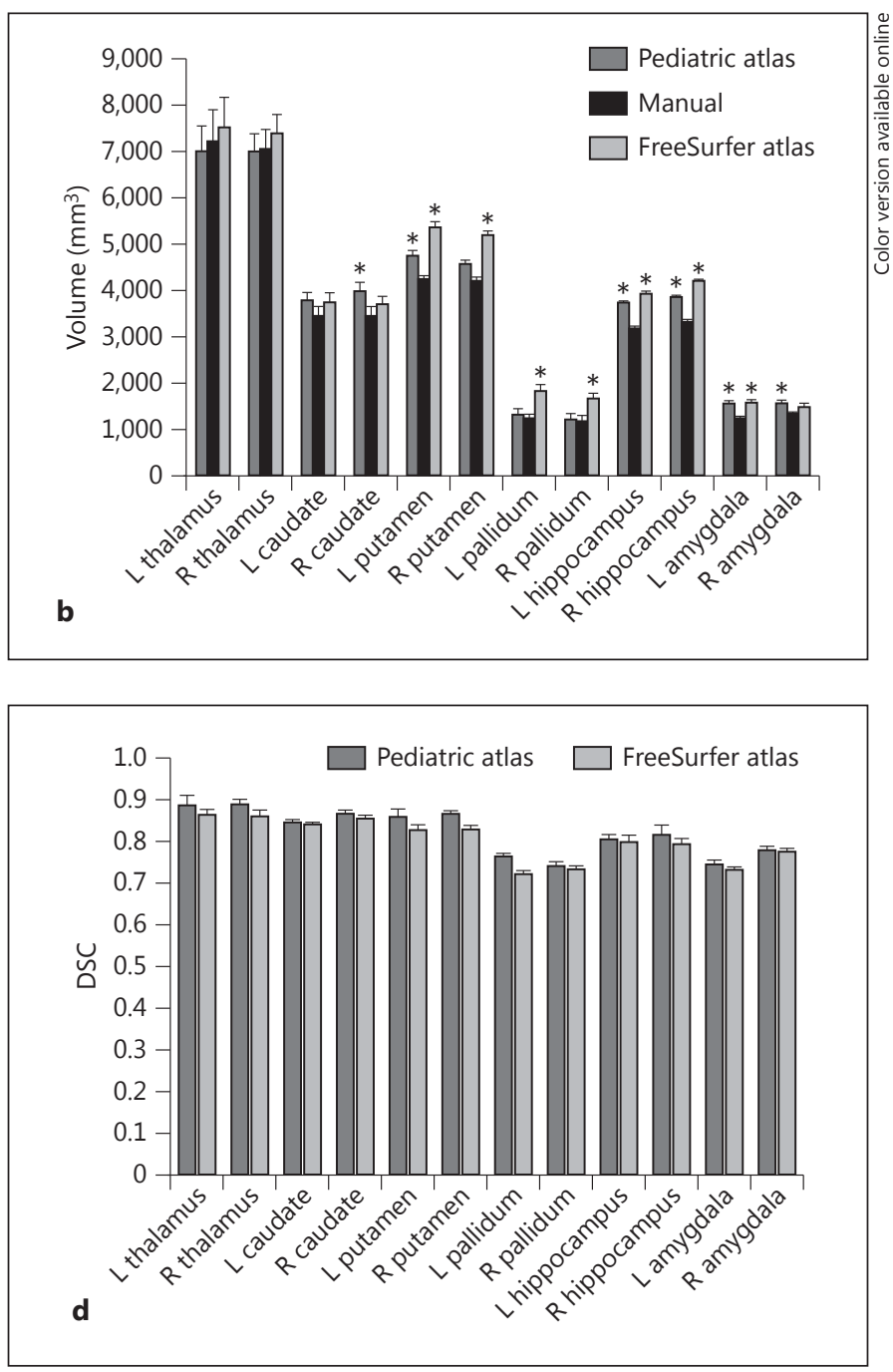

using manual segmentation. ${ }^{*} \mathrm{p}<0.05$ compared with manual segmentation. $\mathbf{c}$ The absolute mean percentage difference between automated FreeSurfer segmentations and manual segmentations of left and right subcortical structures. Automated segmentations were based on a pediatric atlas (in dark gray) or a standard FreeSurfer atlas (in light gray). d DSC as measures of segmentation efficiency of pediatric and standard FreeSurfer atlases for this cohort.

2 groups: CE, and NCE. The CE group consisted of 11 CE children. The NCE group consisted of 10 subjects: 6 children with polysubstance (without cocaine) exposure and 4 children with no substance exposure.

The volumetric differences for each brain structure between the 2 study groups were first assessed without adjustment for other variables. There were 7 female and 4 male children in the CE group and 5 female and 5 male children in the NCE group with matched socioeconomic status (table 1). We also calculated the effect sizes [47] of the regional differences in brain volume. Regression analyses were performed to test the correlations between cocaine as well as other prenatal drug exposure levels within the CE 
Table 1. Subject characteristics in juvenile ( $8-10$ years) and adolescent ( $13-15$ years) cohorts

\begin{tabular}{lll}
$\frac{8-10 \text { years }}{\mathrm{NCE} \quad \mathrm{CE}}$ & & $\frac{13-15 \text { years }}{\mathrm{NCE} \quad \mathrm{CE}}$ \\
\hline
\end{tabular}

\begin{tabular}{lcccc}
\hline Maternal characteristics & & & & \\
Age, years & 25 & 27 & 27 & 28 \\
Alcohol use, \% & 66 & 85 & 65 & 65 \\
Tobacco use, \% & 55 & 71 & 40 & 75 \\
Socioeconomic status & 3.7 & 3.5 & 3.6 & 3.1 \\
\hline
\end{tabular}

\begin{tabular}{lccll}
\hline Infant characteristics & & & & \\
Male, \% & 50 & 63 & 60 & 58 \\
Gestational age, weeks & 38 & 37 & 39 & 38 \\
HC at birth, cm & 33 & 32 & 34 & 34 \\
Length, cm & 49 & 46 & 50 & 49 \\
\hline Child characteristics & & & & \\
Age, years & 8.8 & 8.8 & 13.4 & 13.7 \\
IQ at age of scan & 89 & 88 & 96 & 96 \\
\hline
\end{tabular}

group and the sizes of the brain structures. The regression of the volumes against $\mathrm{CE}$ levels was then adjusted by including exposure levels to other substances in the same linear regression model. Finally, HCs (from 1 month to 9 years old) were compared across groups and tested for their predictive value with respect to the measured morphometric brain alterations. Finally, CE versus NCE group comparisons and time-dependent changes of brain volume estimates of 10 subjects, who were scanned at both ages, were performed using split-plot ANOVA analysis.

\section{Results}

Validity of Using a Pediatric Atlas for Automated

Subcortical Segmentations

Comparing the mean volume of each subcortical structure, we identified that both sets of automated segmentation methods slightly overestimated the volumes of most structures compared with the manual method (fig. 1b), but overall the volumes derived using the pediatric atlas were closer to the manual measures. The percent volume differences between each automated segmentation and the manual segmentation (fig. 1c) further confirms that using the pediatric versus the standard atlas produces volumes closer to the manual results for these subjects. The only exception to this trend is the right caudate, for which the pediatric atlas performance was slightly decreased relative to the standard atlas. Performing a $t$ test across the manual and automated measures shows that there were significant volume differences in the mea- sures for the putamen, pallidum hippocampus and amygdala when the standard FreeSurfer atlas was used. However, the difference between manual and automated segmentation was significant only for the hippocampus and amygdala when the pediatric atlas was used. The DSC reported that there was a good overlap between the automatically and manually segmented areas, especially if the automated segmentation was based on the pediatric atlas (fig. 1d). A good overlap occurs when the DSC is greater than 0.70 , which was met or exceeded for nearly all structures using either atlas. Taken together, all of these measures indicated that the volume estimations were more accurate when the pediatric atlas was used.

\section{Effects of Prenatal CE on Regional Brain \\ Morphometry}

In order to make a comparison across the $\mathrm{CE}$ and the NCE groups, we first examined the cerebral white matter, the cerebral cortex and the subcortical volumes from the 21 subjects. The volumes were derived from automated segmentations using the pediatric atlas that we created comprising the 13 manually segmented data sets.

Initially, we compared the mean volumes associated with individual brain structures in the CE and NCE groups. This comparison revealed a trend towards smaller brain structures in CE children (fig. 2). Significantly smaller volumes were estimated for the cerebral cortex $(\mathrm{p}=0.01$, effect size $=0.85)$ but not for white matter (fig. 2a). Among subcortical structures, significantly smaller volumes were estimated for the thalamus $(\mathrm{p}=$ 0.01 , effect size $=0.77)$ and putamen $(\mathrm{p}=0.04$, effect size $=0.62$; fig. $2 b, c)$. Finally, a similar trend was also observed for intracranial volumes (ICV), but the difference between the groups did not reach statistical significance $(p=0.07)$. Consequently, we repeated the comparison after correcting for the differences in ICV. We found that the regional differences lost significance after correction for ICV, suggesting that the reductions are at least partly attributable to global differences in brain volumes induced by prenatal cocaine exposure (table 2).

\section{Dose-Response Relationships}

In order to assess whether the changes identified in the structural volumes of the cortex, thalamus and putamen additionally depended on the level of CE, we regressed these volume estimates against the levels of maternal substance abuse based on interviews with parents, which were available from 15 ( $7 \mathrm{CE}, 9 \mathrm{NCE}$ ) out of the 21 (11 $\mathrm{CE}, 10 \mathrm{NCE}$ ) participants. Within the CE group, no correlation was found between cerebral cortical volume and 


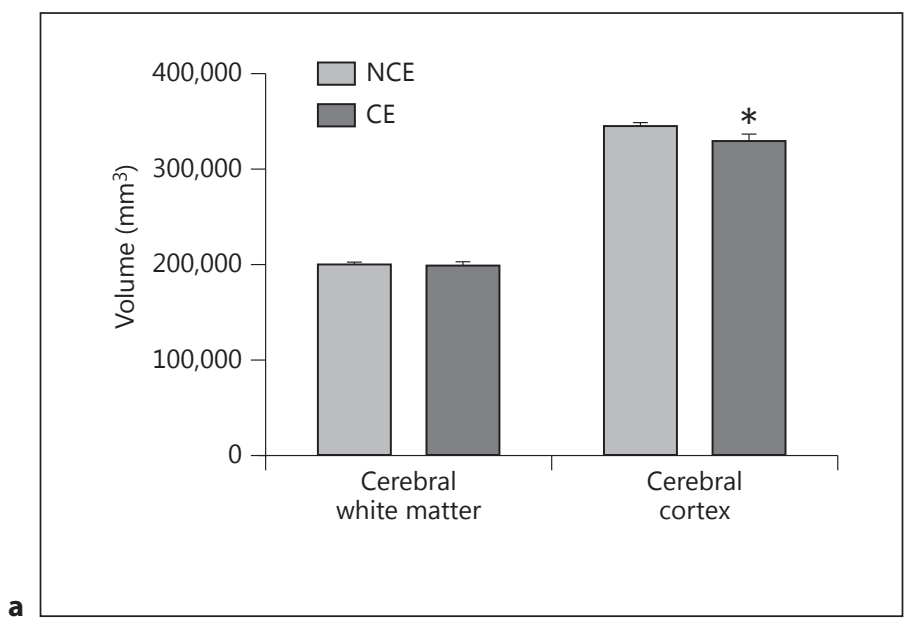

Fig. 2. Volumes of brain structures in CE and NCE children at age 8-10 years. a, b Estimated volumes of cerebral white matter and cortex (a) and of subcortical structures including thalamus, caudate, putamen, pallidum, hippocampus and amygdala (b). NCE and CE group means and standard errors are shown in gray and black, respectively. c Effect sizes and p values for CE $(n=11)$ and NCE $(n=10)$ group comparisons. Significantly different volumes are marked with an asterisk. ${ }^{*} \mathrm{p}<0.05$ compared with controls.

Table 2. Average volume estimates of brain structures in the juvenile cohort ( $8-10$ years; as shown in fig. 2$)$ as well as of ICV

\begin{tabular}{lrrll}
\hline Brain structure & $\begin{array}{l}\text { NCE } \\
\text { volume, } \\
\mathrm{mm}^{3}\end{array}$ & $\begin{array}{l}\text { CE } \\
\text { volume, } \\
\mathrm{mm}^{3}\end{array}$ & $\begin{array}{l}\text { ICV-corrected between- } \\
\text { group comparisons }\end{array}$ \\
\cline { 4 - 5 } & & & & \\
effect sizes & p values \\
Cerebral white & & & \\
matter & 193,872 & 187,865 & 0.33 & 0.31 \\
Cerebral cortex & 338,912 & 316,071 & 0.02 & 0.95 \\
Thalamus & 7,180 & 6,646 & 0.12 & 0.71 \\
Caudate & 4,026 & 3,873 & 0.32 & 0.31 \\
Putamen & 4,767 & 4,441 & 0.05 & 0.88 \\
Pallidum & 1,201 & 1,188 & 0.48 & 0.13 \\
Hippocampus & 3,802 & 3,751 & 0.36 & 0.27 \\
Amygdala & 1,576 & 1,527 & 0.15 & 0.63 \\
Accumbens & 581 & 546 & 0.06 & 0.84 \\
ICV & $1,285,838$ & $1,205,422$ & NA & NA \\
\hline \multicolumn{5}{c}{ ICV-corrected effect sizes and p values are shown. } \\
\hline \multicolumn{5}{c}{} \\
\hline
\end{tabular}

Brain Structures in Children/Adolescents after Prenatal Cocaine Exposure

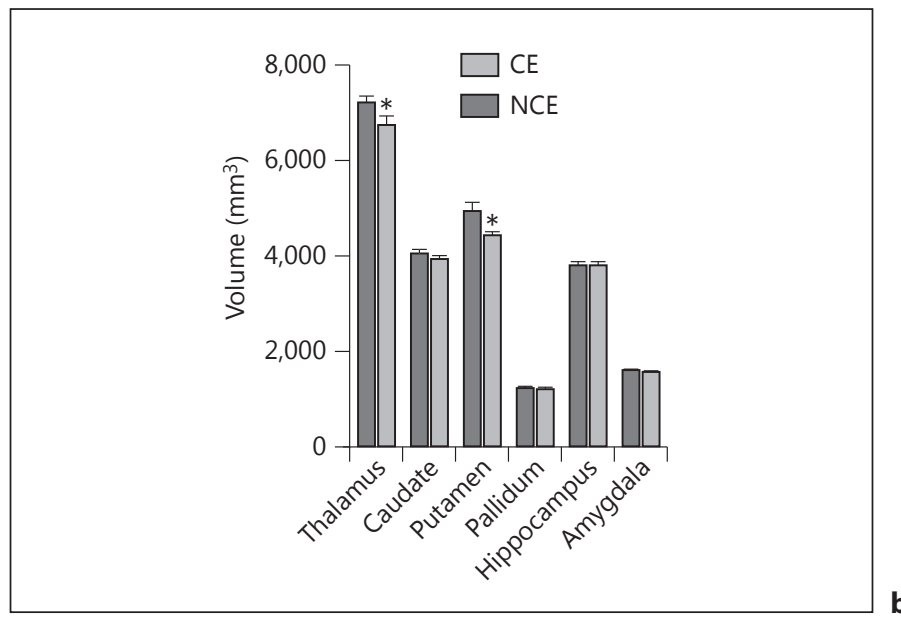

\begin{tabular}{|lll|}
\hline Brain structure & \multicolumn{2}{l|}{$\begin{array}{l}\text { Between-group } \\
\text { comparisons }\end{array}$} \\
\cline { 2 - 2 } & effect sizes & p values \\
\hline Cerebral white matter & 0.27 & 0.39 \\
Cerebral cortex & 0.85 & $0.01^{*}$ \\
Thalamus & 0.77 & $0.01^{*}$ \\
Caudate & 0.42 & 0.17 \\
Putamen & 0.62 & $0.04^{*}$ \\
Pallidum & 0.09 & 0.76 \\
Hippocampus & 0.15 & 0.63 \\
Amygdala & 0.35 & 0.27 \\
\hline
\end{tabular}

Table 3. Regression analysis between structure volumes (dependent variable) and reported exposure levels

a Corrected and uncorrected values for cocaine effects

\begin{tabular}{llllll}
\hline $\begin{array}{l}\text { Brain } \\
\text { structure }\end{array}$ & $\begin{array}{l}\text { Uncorrected } \\
\text { (cocaine) }\end{array}$ & $\begin{array}{l}\text { Corrected } \\
\text { for tobacco }\end{array}$ & $\begin{array}{l}\text { Corrected } \\
\text { for alcohol }\end{array}$ & $\begin{array}{l}\text { Corrected } \begin{array}{l}\text { Corrected } \\
\text { for mari- } \\
\text { juana }\end{array} \\
\text { for coex- } \\
\text { posures }\end{array}$ \\
\hline $\begin{array}{c}\text { Cerebral } \\
\text { cortex }\end{array}$ & 0.08 & 0.79 & 0.47 & 0.89 & 0.35 \\
Thalamus & $0.01^{*}$ & $0.02^{*}$ & $0.02^{*}$ & $0.01^{*}$ & 0.07 \\
Putamen & $0.01^{*}$ & $0.05^{*}$ & $0.05^{*}$ & $0.01^{*}$ & $0.03^{*}$ \\
\hline
\end{tabular}

b Uncorrected values for tobacco, alcohol and marijuana effects

\begin{tabular}{llll}
\hline Brain structure & Tobacco & Alcohol & Marijuana \\
\hline Cerebral cortex & 0.46 & 0.14 & 0.11 \\
Thalamus & 0.08 & 0.08 & 0.24 \\
Putamen & 0.47 & $0.02^{*}$ & 0.28 \\
\hline
\end{tabular}

p values are shown. ${ }^{*} \mathrm{p}<0.05$. 
Fig. 3. Relationship between thalamic and putaminal volumes at age 8-10 years and substance exposure levels. a, b Estimated thalamic and putaminal volumes of $\mathrm{CE}$ children are plotted as a function of cocaine (a) and alcohol exposure levels (b) based on interviews with mothers. c, d Same for ICV. Shown are averages and standard errors of at least 2 independent data points except for CE level 2 (only 1 subject). Solid and dashed lines represent significant and nonsignificant correlations, respectively.

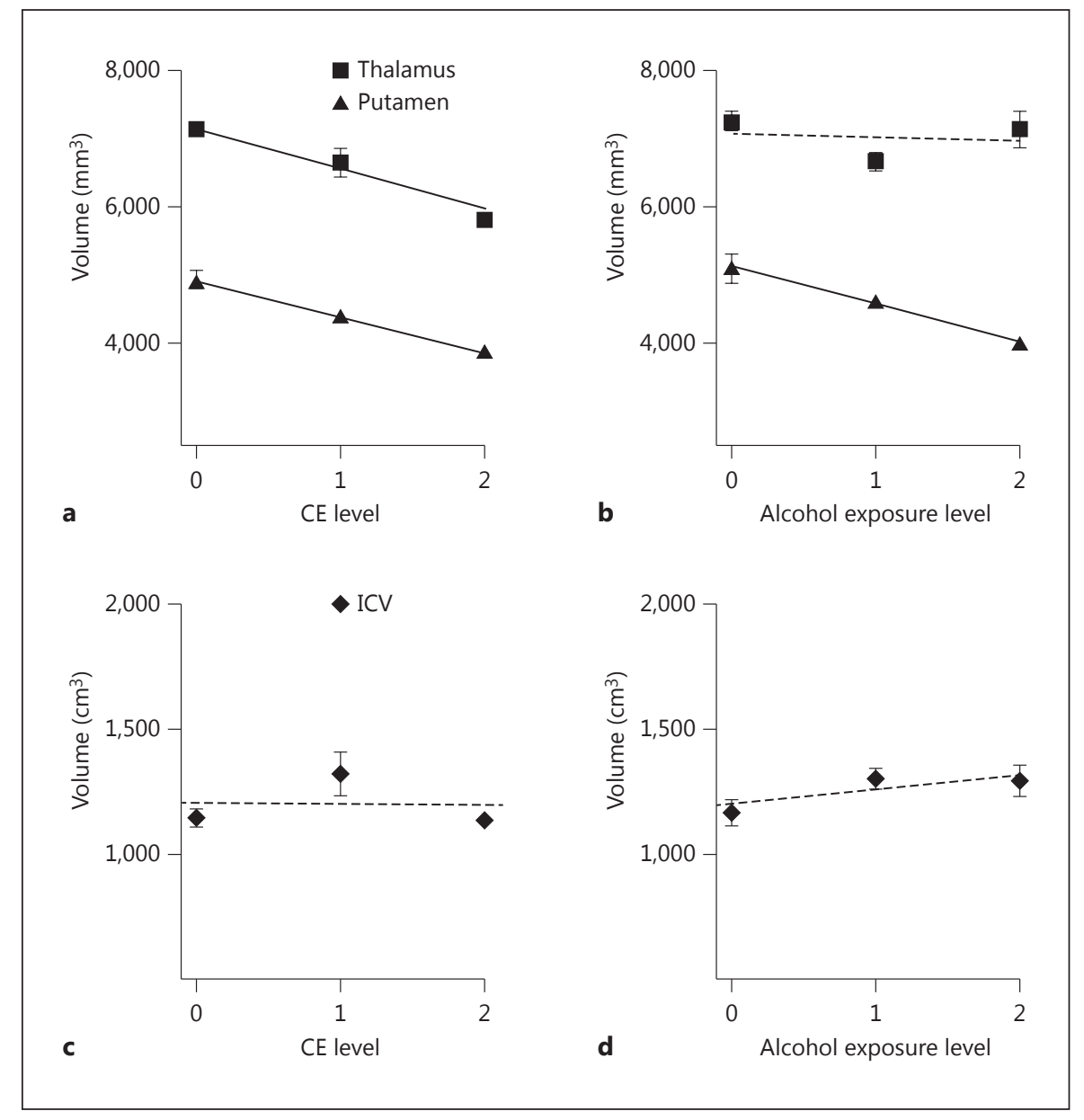

exposure level ( $p=0.8$; table $3 \mathrm{a})$. In contrast, smaller volumes were observed for the thalamus and putamen as a function of CE level (fig. 3a), which points to a robust dose-response relationship specific for these structures (thalamus: $\mathrm{p}<0.01$, putamen: $\mathrm{p}=0.01$ ). Importantly, when the thalamic and putaminal volume regressions against self-reported CE levels were adjusted for self-reported levels of exposure to other substances (alcohol, tobacco, marijuana), it was found that the adjusted $p$ values for both structures in the CE group remained significant (table 3a). However, in the case of putaminal volumes, smaller volumes were observed as a function of prenatal alcohol exposure as well (fig. $3 \mathrm{~b}$; table $3 \mathrm{~b}$ ). The inverse correlation between putaminal volume and alcohol exposure level $(\mathrm{p}<0.05)$, combined with the fact that the effect of alcohol on the putamen remained significant after controlling for $\mathrm{CE}(\mathrm{p}<0.05)$, points to the likelihood of synergistic effects for this structure - both prenatal exposure to alcohol and cocaine significantly decreases putaminal volume. Importantly, these dose-dependent effects were not observed for any other structure (data not shown) or the total ICV (fig. 3c, d).

Relationship between Cortical Volumes at Ages 8-10 and 13-15 Years and HCs

We further explored whether the HCs measured at 1 month, 9 years or 14 years of age could be good predictors of the changes in cortical volumes. Collectively, the HCs of the CE children revealed a trend for smaller sizes compared with those of NCE children from 1 month until 9 years of age (fig. 4), though HCs for both groups fell within 2 standard deviations of the mean (estimated by Center for Disease Prevention Center) [48]. Interestingly, we found that HCs at 9 years of age correlated well with the cortical volumes of both the $\mathrm{CE}$ and $\mathrm{NCE}$ groups at this age (fig. $5 c$, d). On the other hand, the HCs at 1 month of age correlated well with cortical volumes for the CE group but not for the NCE group (fig. 5a, b). 


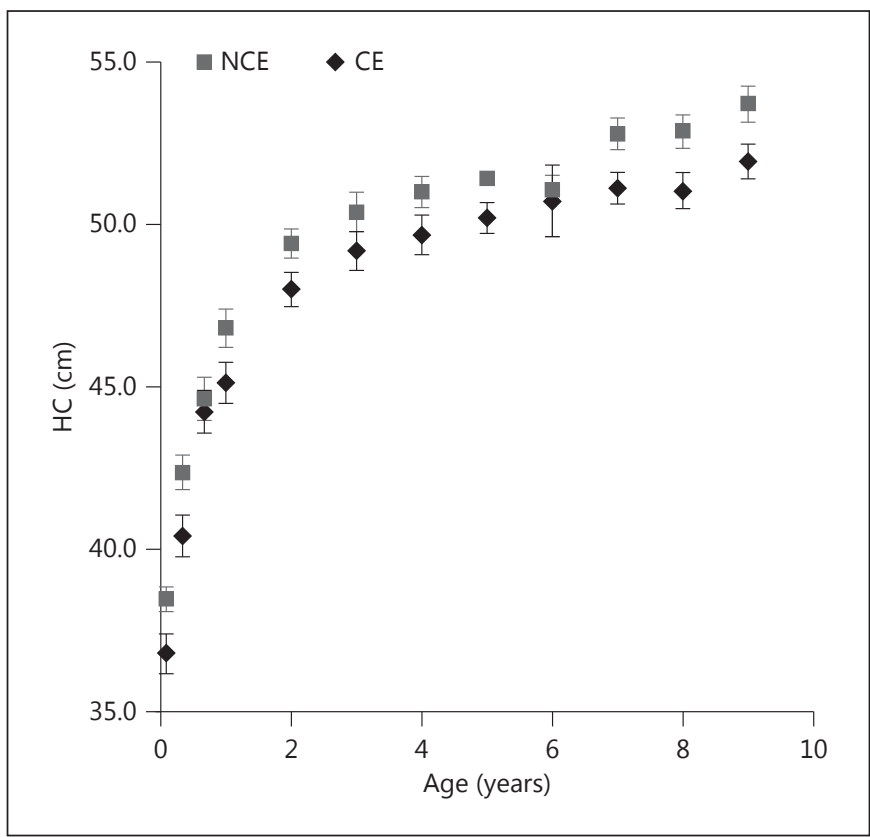

Fig. 4. Longitudinal (from 1 month to 9 years) $\mathrm{HC}$ data for the $\mathrm{CE}$ and NCE cohorts, who were scanned at age 8-10 years. Estimated means and standard errors of HCs in the CE and NCE groups.

Similarly, HCs at 1 month also correlated with the cortical volumes at age 13-15 years in the CE group but not in the NCE group (fig. 5c, d).

\section{Comparison between MRI-Based Brain Volumetrics}

in Juvenile and Adolescent Cohorts

In order to explore how the effect of cocaine on the cortical and subcortical structure volumes depends on age, we compared the brain volumes of the subjects who were scanned at age $8-10$ years with those who were scanned at 13-15 years. The latter group contained 10 subjects (6 CE, $4 \mathrm{NCE}$ ) who were scanned at both time points as well as 30 additional participants (14 CE, $16 \mathrm{NCE}$ ). In contrast with the results reported here for the cohort of children aged 8-10 years, we previously reported that we did not observe differences in cortical, thalamic or putaminal volumes in the cohort of adolescents aged 13-15 years [28]. One possibility for this discrepancy is that there is delayed maturation such that cocaine-induced decrements in the volumes of those structures, which we observed in childhood, may be less evident during adolescence [28]. Consistent with this formulation, a previous report of children aged 7-8 years documented reductions in cortical volume in the CE group [31], while in other studies of CE adoles-
Table 4. Percent growth for CE and NCE subjects who were scanned both at ages $8-10$ and $13-15$ years

\begin{tabular}{llr}
\hline \multirow{2}{*}{ Structure } & \multicolumn{2}{l}{ Growth $(\%)$} \\
\cline { 2 - 3 } & NCE & CE \\
\hline Cerebral white matter & -3 & -11 \\
Cerebral cortex & -8 & -10 \\
Thalamus & -7 & -7 \\
Caudate & -1 & -4 \\
Putamen & 8 & -1 \\
Pallidum & -1 & 6 \\
Hippocampus & 9 & 1 \\
Amygdala & 24 & 7 \\
Accumbens & 44 & 31 \\
ICV & 18 & 12 \\
\hline
\end{tabular}

cents aged 10-14 years such effects were either not observed [49] or were attributable to other factors [26]. However, another potential explanation is that the juvenile and adolescent cohorts we studied were different in some other way, perhaps due to factors resulting from the different selection factors utilized to identify the 2 cohorts. In order to consider both of these possibilities, we first examined how the brain volumes changed in individual subjects who were scanned both at age 8-10 and 13-15 years. Within this group of subjects scanned twice $(\mathrm{n}=10)$, we found a trend for smaller brain structures at both ages, reaching statistical significance only for the cortex (fig. 6b). Importantly, during the time interval between 8-10 and 13-15 years, the cortical, thalamic and putaminal volumes showed comparable changes for the CE and NCE groups (fig. 6a; table 4), suggesting the likelihood that these differences are not due to a group-specific delay in maturation. Here, we also note that the observed reduction in volumes of cortical and subcortical structures over the 5 -year interval we studied is largely consistent with previous studies also reporting larger structures in school-aged children compared with young adults [36, 50-52]. Furthermore, when we compared the average brain volumes across sexes, we found that for most brain structures the female brain was estimated to be about $90 \%$ of that of the male brain at both ages (table 5). This is consistent with previous findings reported by others during normal brain development [36] and excludes the possibility that CE differentially altered brain growth in our subjects in a sexually dimorphic manner. Subsequently, we compared the juvenile and adolescent cohorts with respect to demographic and clinical variables. We found no obvious dif- 
Fig. 5. Relationship between cortical volumes at age $8-10$ years and HCs. Estimated cerebral cortical volumes at age $8-10$ years are plotted as a function of HCs at 1 month $(\mathbf{a}, \mathbf{b})$ and at age 9 years $(\mathbf{c}, \mathbf{d})$ for $\mathrm{CE}(\mathbf{a}, \mathbf{c})$ and NCE $(\mathbf{b}, \mathbf{d})$ groups, respectively.

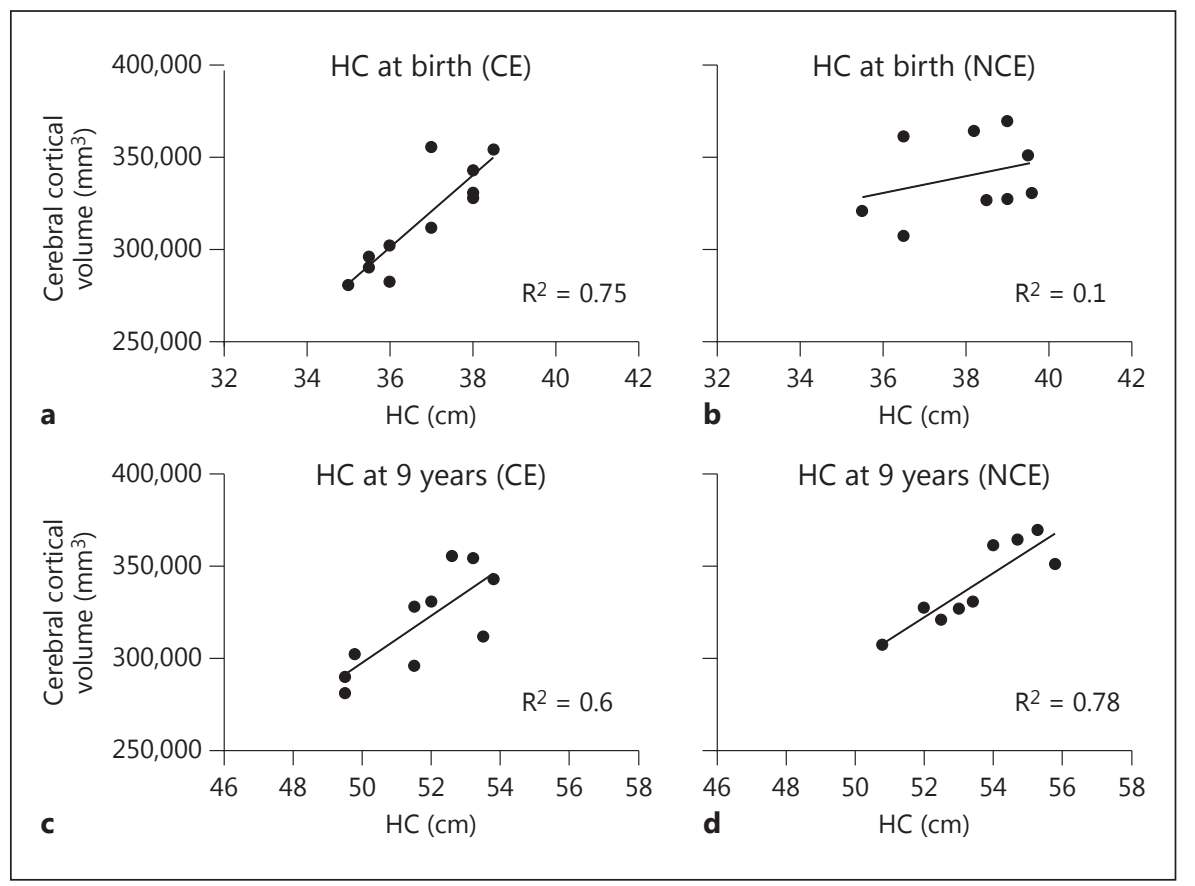

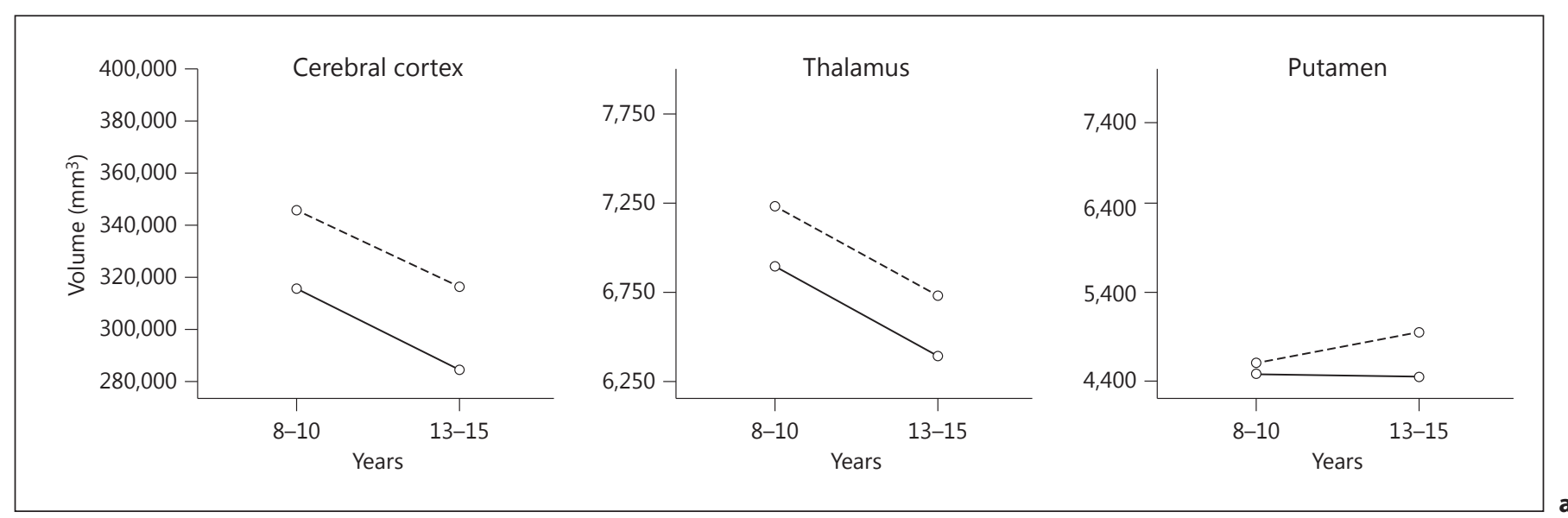

Fig. 6. Growth patterns from age 8-10 to 13-15 years. a Means and standard errors of volume estimates of the cerebral cortex, thalamus and putamen in subjects scanned at 2 different ages. CE ( $\mathrm{n}=$ $6)$ and NCE $(n=4)$ children are represented with solid and dashed lines, respectively. Time 1 represents children aged 8-10 years, while time 2 represents subjects at $13-15$ years. b p values for CE vs. NCE group comparisons of brain volume estimates of subjects who were scanned at both ages. Significantly different $\mathrm{p}$ values are marked with an asterisk. ${ }^{*} \mathrm{p}<0.05$ compared with controls.

\begin{tabular}{|llll|}
\hline Brain structure & \multicolumn{3}{l}{ Group comparisons ( $\mathrm{p}$ value) } \\
\cline { 2 - 4 } & $\begin{array}{l}\text { 8-10 } \\
\text { years }\end{array}$ & $\begin{array}{l}13-15 \\
\text { years }\end{array}$ & $\begin{array}{l}\text { change over } \\
\text { time }\end{array}$ \\
\hline Cerebral white matter & 0.6 & 0.122 & $0.02^{*}$ \\
Cerebral cortex & $0.02^{*}$ & $0.02^{*}$ & 0.61 \\
Thalamus & 0.28 & 0.45 & 0.99 \\
Caudate & 0.4 & 0.09 & 0.20 \\
Putamen & 0.57 & 0.16 & 0.32 \\
Pallidum & 0.84 & 0.49 & 0.51 \\
Hippocampus & 0.36 & 0.18 & $0.02^{*}$ \\
Amygdala & 0.56 & 0.004 & $0.02^{*}$ \\
\end{tabular}




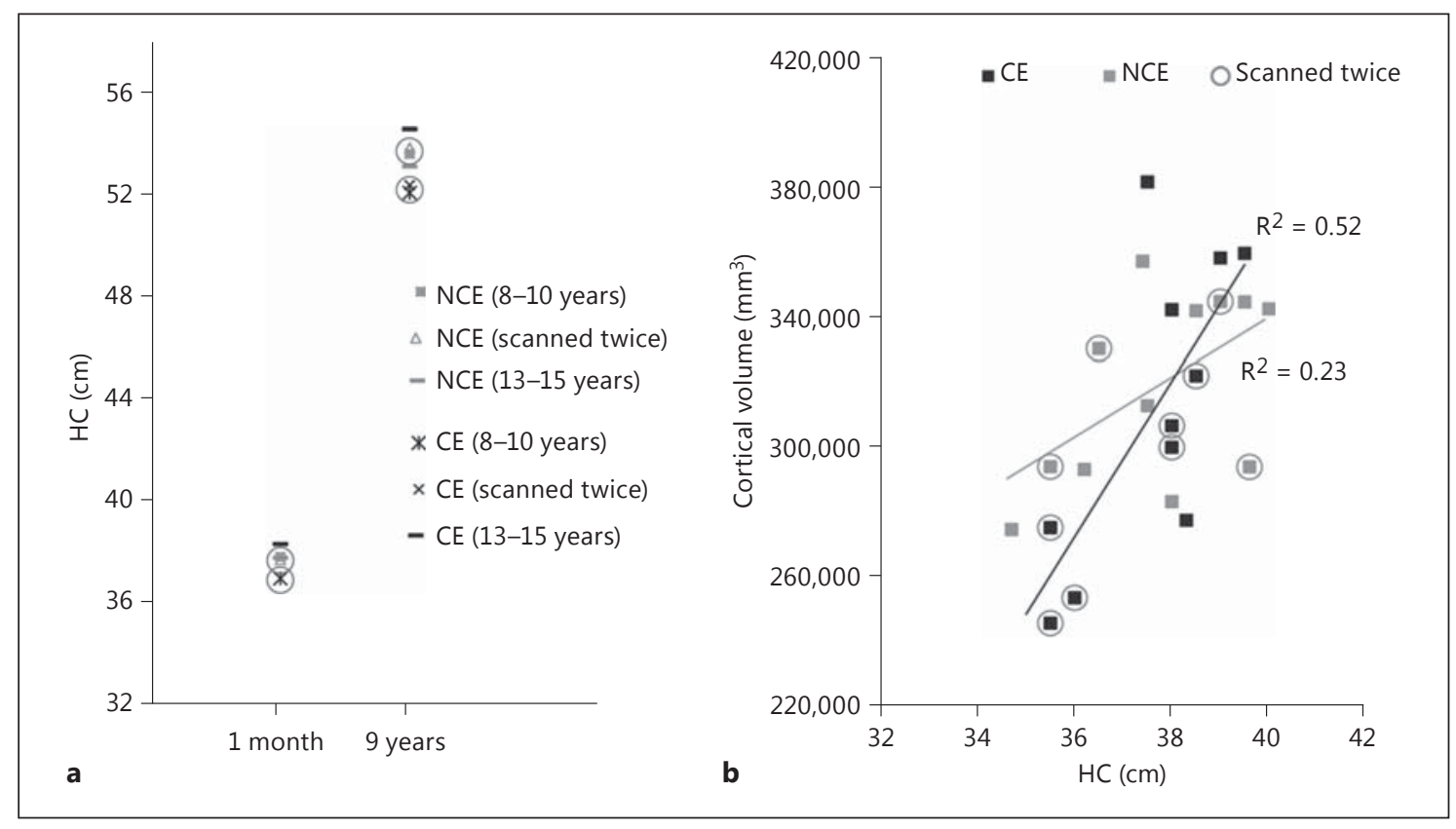

Fig. 7. HCs at birth are predictive of cerebral cortical volume in the CE but not the NCE group. a Average HCs at age 1 month in CE and NCE groups for the cohort scanned at age 8-10 years, for the cohort scanned only at age 13-15 years, and for the cohort scanned at both ages. b Cerebral cortical volumes at age 13-15 years as a function of HCs at age 1 month for the CE and NCE groups shown in black and gray squares, respectively. Values for the individuals scanned twice are circled in both plots.

Table 5. Ratio of female-to-male brain volumes at ages $8-10$ and $13-15$ years

\begin{tabular}{lll}
\hline Structure & $8-10$ years & $13-15$ years \\
\hline White matter & 0.92 & 0.86 \\
Cortex & 0.91 & 0.86 \\
Thalamus & 0.97 & 0.86 \\
Caudate & 1.01 & 0.98 \\
Putamen & 0.94 & 0.90 \\
Pallidum & 0.95 & 0.93 \\
Hippocampus & 0.93 & 0.92 \\
Amygdala & 0.93 & 0.87 \\
Accumbens & 0.86 & 1.00 \\
ICV & 0.87 & 0.87 \\
\hline
\end{tabular}

ferences in the demographic profiles of the CE and NCE groups (data not shown). However, we did confirm that the adolescent cohort was different from the juvenile cohort, which was matched for HCs in advance by study design [28]. Specifically, we found that for the adolescent cohort newly recruited subjects in the CE group had relatively larger mean HCs at 1 month of age (fig. 7a) and consequently larger brain structures [28], which may have precluded identification of the group effects in the older CE cohort studied. Consistent with this hypothesis, a strong correlation was observed between HCs at 1 month of age and cerebral cortical volumes at age 13-15 years in the CE group but not in the NCE group (fig. 7b; table 6), as was seen at age 8-10 years in the cohort we studied (fig. 5a, b). Despite the modest sample size for the individuals scanned twice, these results point to the validity of the specific changes in HCs and brain volumes we have identified in the CE group of children aged 8-10 years.

\section{Discussion}

In this study, we determined the volumes of subcortical brain structures in children aged 8-10 years from the MLS cohort. The pediatric atlas that we developed provided greater validity for segmenting brain structures than the standard FreeSurfer adult atlas. We reported mean percent differences, DSC and Hausdorff distances that demonstrated that the volume estimates were closer to those from manual segmentations when the pediatric atlas was utilized instead of the standard FreeSurfer atlas. The validity of using this pediatric atlas, or a more com- 
Table 6. Linear regression analysis of HCs (independent variable) at birth with cerebral cortical volumes (dependent variable) at ages $8-10$ and $13-15$ years

\begin{tabular}{rcc}
\hline & NCE & CE \\
\hline $8-10$ years & 0.63 & $<0.01^{*}$ \\
$13-15$ years & 0.09 & $0.01^{*}$ \\
\hline
\end{tabular}

p values are shown. ${ }^{*} \mathrm{p}<0.05$.

prehensive version based on a larger number of scans, needs to be further confirmed using images acquired with other MRI pulse sequences and on different MRI scanners. Recently improved versions of FreeSurfer have incorporated additional steps to normalize the range of MRI signal intensities and employ within-subject templates in longitudinal studies [53]. These upgrades in the FreeSurfer software will help minimize the variance introduced by using different MRI data acquisition schemes and different scanners, further contributing to the 'value added' of using a pediatric atlas for developmental morphometric studies.

In the cohort aged 8-10 years, we observed region-specific reductions in the volume of the cerebral cortex, thalamus and putamen in the CE group. However, it is difficult to identify whether or not these effects are directly linked to prenatal CE for each of these regions. For the CE group, cerebral cortical volume did not show a dose-response relationship when regressed against self-reported levels of CE. However, for the CE group thalamic and putaminal volumes revealed a dose-response relationship when regressed against self-reported levels of CE. Of note, a correlation between putaminal volume and alcohol exposure was observed for all subjects who self-reported their levels of alcohol intake, suggesting that for this structure the effects of CE may be additive to those of alcohol exposure, especially at high doses of both. Given our small cohort comprised of many polysubstance-exposed children, it is difficult to separate the effects. However, by including 10 polysubstance-exposed (but not CE) children in our control group of 15 subjects, we designed our study to independently identify the additional consequences of prenatal CE. Thus, our study points towards a reduction of cortical, thalamic and putaminal volumes in school-aged children (8-10 years) as being in part attributable to prenatal CE.

Others have reported a prenatal cocaine-induced volume reduction in the caudate in children as well as in adolescents $[26,30]$. In our cohort, we also observed a tendency for a smaller caudate; however, the group differences did reach not statistical significance either before or after correcting for ICV (fig. 2; table 2).

$\mathrm{HC}$ is commonly used as an indicator of brain growth during infancy and early childhood, a time during which facial growth contributes less to HC. However, the relationship between $\mathrm{HC}$ and brain volume is dynamic and changes throughout the life span. Throughout early childhood, both HCs and brain volumes increase and from late adolescence onwards brain volumes start decreasing, while HCs do not [54]. Interestingly, in the NCE group we found that HCs at 9 years of age correlated well with cortical volumes at age 8-10 years, but HCs at 1 month of age did not. For the CE group, HCs at both 1 month and 9 years of age did show a correlation with the cortical volumes at age 8-10 years. This suggests that for a subset of this group, prenatal CE compromises fetal brain growth, as reflected by relatively smaller HCs at 1 month, with implications for poor brain growth that remain evident for at least 8-10 years. However, specific effects of confounding factors, including other prenatal insults, as well as additional genetic and postnatal environmental factors in contributing to impaired postnatal brain growth following prenatal exposure to drugs of abuse need to be identified in a larger cohort [for example, see 55]. Towards this goal, multi-institutional (e.g. PING: http://pingstudy. ucsd.edu) and multinational (e.g. IMAGEN: http://www. imagen-europe.com/en/consortium.php) efforts to facilitate the acquisition of larger-scale and standardized data collection during childhood and adolescence are now underway to integrate MRI-based structural and functional imaging parameters with other measures including demographic information, genetic analyses, clinical evaluations and neuropsychological assessments, thereby providing a more comprehensive appreciation of factors contributing to normal versus abnormal brain development.

\section{Acknowledgments}

This work was in part supported by NIH grants DA000354 and DA17905 (B.E.K.) and U10-DA-024119-01, U10-HD-27904 (to BML), NICHD contract N01-HD-2-3159 (to BML), and NIH R01 MH083320 and P30 HD004147 (D.K.).

\section{Disclosure Statement}

The authors have no conflicts of interest to disclose with this paper. 


\section{References}

$\checkmark 1$ Lidow MS, Song ZM: Primates exposed to co- 16 Stanwood GD, Washington RA, Shumsky JS, caine in utero display reduced density and number of cerebral cortical neurons. J Comp Neurol 2001;435:263-275.

$>2$ Kosofsky BE, Wilkins AS, Gressens P, Evrard P: Transplacental cocaine exposure: a mouse model demonstrating neuroanatomic and behavioral abnormalities. J Child Neurol 1994; 9:234-241.

$\checkmark 3$ Volpe JJ: Effect of cocaine use on the fetus. N Engl J Med 1992;327:399-407.

4 Kapur RP, Shaw CM, Shepard TH: Brain hemorrhages in cocaine-exposed human fetuses. Teratology 1991;44:11-18.

$\checkmark 5$ Woods JR Jr, Plessinger MA, Clark KE: Effect of cocaine on uterine blood flow and fetal oxygenation. JAMA 1987;257:957-961.

-6 Meyer JS, Shearman LP, Collins LM, Maguire RL: Cocaine binding sites in fetal rat brain: implications for prenatal cocaine action. Psychopharmacology (Berl) 1993;112:445-451.

-7 Koegler SM, Seidler FJ, Spencer JR, Slotkin TA: Ischemia contributes to adverse effects of cocaine on brain development: suppression of ornithine decarboxylase activity in neonatal rat. Brain Res Bull 1991;27:829-834.

$>8$ Ritz MC, Lamb RJ, Goldberg SR, Kuhar MJ: Cocaine receptors on dopamine transporters are related to self-administration of cocaine. Science 1987;237:1219-1223.

$\checkmark 9$ Bergman J, Madras BK, Johnson SE, Spealman RD: Effects of cocaine and related drugs in nonhuman primates. III. Self-administration by squirrel monkeys. J Pharmacol Exp Ther 1989;251:150-155.

-10 Kuhar MJ, Ritz MC, Boja JW: The dopamine hypothesis of the reinforcing properties of cocaine. Trends Neurosci 1991;14:299-302.

$>11$ Mayes LC: A behavioral teratogenic model of the impact of prenatal cocaine exposure on arousal regulatory systems. Neurotoxicol Teratol 2002;24:385-395.

12 Liu J, Lester BM: Reconceptualizing in a dualsystem model the effects of prenatal cocaine exposure on adolescent development: a short review. Int J Dev Neurosci 2011;29:803-809.

13 Glatt SJ, Bolanos CA, Trksak GH, Jackson D: Effects of prenatal cocaine exposure on dopamine system development: a meta-analysis. Neurotoxicol Teratol 2000;22:617-629.

-14 Tropea TF, Guerriero RM, Willuhn I, Unterwald EM, Ehrlich ME, Steiner H, Kosofsky BE: Augmented $\mathrm{D}_{1}$ dopamine receptor signaling and immediate-early gene induction in adult striatum after prenatal cocaine. Biol Psychiatry 2008;63:1066-1074.

-15 Kabir ZD, Lourenco F, Byrne ME, Katzman A, Lee F, Rajadhyaksha AM, Kosofsky BE: Brain-derived neurotrophic factor genotype impacts the prenatal cocaine-induced mouse phenotype. Dev Neurosci 2012;34:184-197. Levitt P: Prenatal cocaine exposure produces consistent developmental alterations in dopamine-rich regions of the cerebral cortex. Neuroscience 2001;106:5-14

17 Singer LT, Arendt R, Minnes S, Farkas K, Salvator A, Kirchner HL, Kliegman R: Cognitive and motor outcomes of cocaine-exposed infants. JAMA 2002;287:1952-1960.

18 Eyler FD, Behnke M, Conlon M, Woods NS, Wobie K: Birth outcome from a prospective, matched study of prenatal crack/cocaine use. II. Interactive and dose effects on neurobehavioral assessment. Pediatrics 1998;101: 237-241.

19 Singer LT, Salvator A, Arendt R, Minnes S, Farkas K, Kliegman R: Effects of cocaine/ polydrug exposure and maternal psychological distress on infant birth outcomes. Neurotoxicol Teratol 2002;24:127-135.

20 Lester BM, LaGasse LL, Seifer R: Cocaine exposure and children: the meaning of subtle ef fects. Science 1998;282:633-634.

21 Bada HS, Das A, Bauer CR, Shankaran S, Lester B, LaGasse L, Hammond J, Wright LL, Higgins R: Impact of prenatal cocaine exposure on child behavior problems through school age. Pediatrics 2007;119:e348-e359.

22 Singer LT, Minnes S, Short E, Arendt R, Farkas K, Lewis B, Klein N, Russ S, Min MO, Kirchner HL: Cognitive outcomes of preschool children with prenatal cocaine exposure. JAMA 2004;291:2448-2456.

23 Bandstra ES, Vogel AL, Morrow CE, Xue L, Anthony JC: Severity of prenatal cocaine exposure and child language functioning through age seven years: a longitudinal latent growth curve analysis. Subst Use Misuse 2004;39:25-59.

24 Bada HS, Das A, Bauer CR, Shankaran S, Lester B, Wright LL, Verter J, Smeriglio VL, Finnegan LP, Maza PL: Gestational cocaine exposure and intrauterine growth: Maternal Lifestyle Study. Obstet Gynecol 2002;100: 916-924.

25 Bateman DA, Chiriboga CA: Dose-response effect of cocaine on newborn head circumference. Pediatrics 2000;106:E33.

26 Rivkin MJ, Davis PE, Lemaster JL, Cabral HJ, Warfield SK, Mulkern RV, Robson CD, RoseJacobs R, Frank DA: Volumetric MRI study of brain in children with intrauterine exposure to cocaine, alcohol, tobacco, and marijuana. Pediatrics 2008;121:741-750.

27 Lester BM, Tronick EZ, LaGasse L, Seifer R, Bauer CR, Shankaran S, Bada HS, Wright LL, Smeriglio VL, Lu J, Finnegan LP, Maza PL: The Maternal Lifestyle Study: effects of substance exposure during pregnancy on neurodevelopmental outcome in 1-month-old in fants. Pediatrics 2002;110:1182-1192.
28 Liu J, Lester BM, Neyzi N, Sheinkopf SJ, Gracia L, Kekatpure M, Kosofsky BE: Regional brain morphometry and impulsivity in adolescents following prenatal exposure to cocaine and tobacco. JAMA Pediatr 2013;167: 348-354.

29 Liu J, Cohen RA, Gongvatana A, Sheinkopf SJ, Lester BM: Impact of prenatal exposure to cocaine and tobacco on diffusion tensor imaging and sensation seeking in adolescents. J Pediatr 2011;159:771-775.

30 Rao H, Wang J, Giannetta J, Korczykowski M, Shera D, Avants BB, Gee J, Detre JA, Hurt H: Altered resting cerebral blood flow in adolescents with in utero cocaine exposure revealed by perfusion functional MRI. Pediatrics 2007 ; 120:e1245-e1254.

31 Dow-Edwards DL, Benveniste H, Behnke M, Bandstra ES, Singer LT, Hurd YL, Stanford LR: Neuroimaging of prenatal drug exposure. Neurotoxicol Teratol 2006;28:386-402.

-32 Avants BB, Hurt H, Giannetta JM, Epstein CL, Shera DM, Rao H, Wang J, Gee JC: Effects of heavy in utero cocaine exposure on adolescent caudate morphology. Pediatr Neurol 2007;37:275-279.

33 Shankaran S, Bauer CR, Bada HS, Lester BM, Wright LL, Katsikiotis V: Maternal Lifestyle Study: patterns of cocaine use in term pregnancy and effect on birthweight. Pediatr Res 1996;39:279A.

-34 Fischl B: FreeSurfer. Neuroimage 2012;62: 774-781.

35 Filipek PA, Kennedy DN, Caviness VS Jr, Rossnick SL, Spraggins TA, Starewicz PM: Magnetic resonance imaging-based brain morphometry: development and application to normal subjects. Ann Neurol 1989;25:6167.

36 Caviness VS Jr, Kennedy DN, Richelme C, Rademacher J, Filipek PA: The human brain age 7-11 years: a volumetric analysis based on magnetic resonance images. Cereb Cortex 1996;6:726-736.

-37 Kennedy DN, Filipek PA, Caviness VR: Anatomic segmentation and volumetric calculations in nuclear magnetic resonance imaging. IEEE Trans Med Imaging 1989;8:1-7.

38 Desikan RS, Segonne F, Fischl B, Quinn BT Dickerson BC, Blacker D, Buckner RL, Dale AM, Maguire RP, Hyman BT, Albert MS, Killiany RJ: An automated labeling system for subdividing the human cerebral cortex on MRI scans into gyral-based regions of interest. Neuroimage 2006;31:968-980.

39 Fischl B, Salat DH, Busa E, Albert M, Dieterich M, Haselgrove C, van der Kouwe A, Killiany R, Kennedy D, Klaveness S, Montillo A, Makris N, Rosen B, Dale AM: Whole brain segmentation: automated labeling of neuroanatomical structures in the human brain. Neuron 2002;33:341-355. 
40 Fischl B, van der Kouwe A, Destrieux C, Halgren E, Segonne F, Salat DH, Busa E, Seidman LJ, Goldstein J, Kennedy D, Caviness V, Makris N, Rosen B, Dale AM: Automatically parcellating the human cerebral cortex. Cereb Cortex 2004;14:11-22.

-41 Buckner RL, Head D, Parker J, Fotenos AF, Marcus D, Morris JC, Snyder AZ: A unified approach for morphometric and functional data analysis in young, old, and demented adults using automated atlas-based head size normalization: reliability and validation against manual measurement of total intracranial volume. Neuroimage 2004;23:724738.

-42 Seidman LJ, Faraone SV, Goldstein JM, Goodman JM, Kremen WS, Toomey R, Tourville J, Kennedy D, Makris N, Caviness VS, Tsuang MT: Thalamic and amygdala-hippocampal volume reductions in first-degree relatives of patients with schizophrenia: An MRI-based morphometric analysis. Biol Psychiatry 1999;46:941-954.

43 Efron B: The Jackknife, the Bootstrap and Other Resampling Plans. Philadelphia, SIAM, 1982.
44 Efron B, Tibshirani RJ: An Introduction to the Bootstrap. London, Chapman \& Hall, 1993.

45 Dice LR: Measures of the amount of ecologic association between species. Ecology 1945;26: 297-302.

46 Huttenlocher DP, Klanderman GA, Rucklidge WJ: Comparing images using the Hausdorff distance. IEEE Trans Pattern Anal Mach Intell 1993:850-863.

47 Cohen J: Statistical Power Analysis for the Behavioral Sciences. Hillsdale, Lawrence Erlbaum, 1988.

48 Kuczmarski RJ, Ogden CL, Grummer-Strawn LM, Flegal KM, Guo SS, Wei R, Mei Z, Curtin LR, Roche AF, Johnson CL: CDC growth charts: United States. Adv Data 2000;314:127.

49 Roussotte F, Soderberg L, Warner T, Narr K, Lebel C, Behnke M, Davis-Eyler F, Sowell E: Adolescents with prenatal cocaine exposure show subtle alterations in striatal surface morphology and frontal cortical volumes. J Neurodev Disord 2012;4:22.

50 Pfefferbaum A, Mathalon DH, Sullivan EV, Rawles JM, Zipursky RB, Lim KO: A quantitative magnetic resonance imaging study of changes in brain morphology from infancy to late adulthood. Arch Neurol 1994;51:874887.
51 Jernigan TL, Tallal P: Late childhood changes in brain morphology observable with MRI. Dev Med Child Neurol 1990;32:379-385.

52 Giedd JN, Blumenthal J, Jeffries NO, Castellanos FX, Liu H, Zijdenbos A, Paus T, Evans AC, Rapoport JL: Brain development during childhood and adolescence: a longitudinal MRI study. Nat Neurosci 1999;2:861-863.

53 Reuter M, Schmansky NJ, Rosas HD, Fischl B: Within-subject template estimation for unbiased longitudinal image analysis. Neuroimage 2012;61:1402-1418.

54 Bartholomeusz HH, Courchesne E, Karns CM: Relationship between head circumference and brain volume in healthy normal toddlers, children, and adults. Neuropediatrics 2002;33:239-241.

55 Lotfipour S, Ferguson E, Leonard G, Perron M, Pike B, Richer L, Seguin JR, Toro R, Veillette S, Pausova Z, Paus T: Orbitofrontal cortex and drug use during adolescence: role of prenatal exposure to maternal smoking and BDNF genotype. Arch Gen Psychiatry 2009; 66:1244-1252. 